Accepted to the Astrophysical Journal, September 12, 2006

Preprint typeset using LATEX style emulateapj v. 6/22/04

\title{
SLOW STAR FORMATION IN DENSE GAS: EVIDENCE AND IMPLICATIONS
}

\author{
MARK R. KRUMHOLZ* \\ Department of Astrophysical Sciences, Princeton University, Princeton, NJ 08544
}

JONATHAN C. TAN

Department of Astronomy, University of Florida, Gainesville, FL 32611

Accepted to the Astrophysical Journal, September 12, 2006

\begin{abstract}
It has been known for more than 30 years that star formation in giant molecular clouds (GMCs) is slow, in the sense that only $\sim 1 \%$ of the gas forms stars every free-fall time. This result is entirely independent of any particular model of molecular cloud lifetime or evolution. Here we survey observational data on higher density objects in the interstellar medium, including infrared dark clouds and dense molecular clumps, to determine if these objects form stars slowly like GMCs, or rapidly, converting a significant fraction of their mass into stars in one free-fall time. We find no evidence for a transition from slow to rapid star formation in structures covering three orders of magnitude in density. This has important implications for models of star formation, since competing models make differing predictions for the characteristic density at which star formation should transition from slow to rapid. The data are inconsistent with models that predict that star clusters form rapidly and in free-fall collapse. Magnetic- and turbulence-regulated star formation models can reproduce the observations qualitatively, and the turbulence-regulated star formation model of Krumholz \& McKee quantitatively reproduces the infrared-HCN luminosity correlation recently reported by Gao \& Solomon. Slow star formation also implies that the process of star cluster formation cannot be one of global collapse, but must instead proceed over many free-fall times. This suggests that turbulence in star-forming clumps must be driven, and that the competitive accretion mechanism does not operate in typical cluster-forming molecular clumps.

Subject headings: ISM: clouds — stars: formation
\end{abstract}

\section{INTRODUCTION}

Zuckerman \& Evans (1974) first pointed out that star formation in giant molecular clouds (GMCs) happens surprisingly slowly. Comparing the mass of GMCs in the Galaxy with the total Galactic star formation rate implies that no more than $\sim 1 \%$ of the gas can form stars for each cloud free-fall time. This result is sufficiently surprising that numerous theories have been proposed to explain it, ranging from the idea that strong magnetic fields (e.g. Allen \& Shu 2000) or turbulence (e.g. Krumholz \& McKee 2005) within clouds inhibit star formation to the idea that galactic-scale gravitational instability regulates star formation (e.g. Li et al. 2005a) to the idea that GMCs are, contrary to most observational estimates to date (Blitz et al. 2005), actually gravitationally unbound (e.g. Clark \& Bonnell 2004).

Following Krumholz \& McKee (2005), we define the dimensionless star formation rate per free-fall time $\mathrm{SFR}_{\mathrm{ff}}$ as the fraction of an object's mass that it converts into stars per free-fall time at the mean density of that object. The Zuckerman \& Evans (1974) argument shows that $\mathrm{SFR}_{\mathrm{ff}} \approx 0.01$ for GMCs. This provides a powerful constraint on models of GMCs. For example, it rules out the early GMC model of Goldreich \& Kwan (1974), a simplified version of which is that GMCs are spheres of gas of density $\rho$ that are in free-fall collapse. The clouds reach a singularity in a time $t_{\mathrm{ff}}(\rho)=[3 \pi /(32 G \rho)]^{1 / 2}$, at which point their mass is converted into stars. For a pop-

${ }^{*}$ Hubble Fellow ulation of such clouds $\mathrm{SFR}_{\mathrm{ff}}=1$, which is inconsistent with the Zuckerman \& Evans (1974) result.

An important observational question, and a crucial test for theories of how star formation is regulated, is to what densities and length scales $\mathrm{SFR}_{\mathrm{ff}}$ remains much smaller than unity. In other words, is there a density at which something like the Goldreich \& Kwan (1974) free-fall collapse model becomes reasonable? As an example, consider observing a star-forming region using a molecular tracer sensitive to gas at densities of $n_{H} \gtrsim 10^{12}$ $\mathrm{cm}^{-3}$, where $n_{H}$ is the number density of hydrogen nuclei, to estimate the total mass of gas at such high densities. This is larger than the mean density of "cores" seen both observationally (e.g. Barranco \& Goodman 1998) and in simulations of star formation regulated by turbulence (e.g. Jappsen et al. 2005), and is roughly the density at which models of magnetically-regulated star formation predict that gas will completely decouple from the magnetic field and enter free-fall collapse (e.g. Desch \& Mouschovias 2001). At such high densities protostellar outflows can probably stop at most half the gas from reaching a star (Matzner \& McKee 2000), and the thermal pressure in gas at $10^{12} \mathrm{~cm}^{-3}$ is considerably higher than the typical ram pressure of the turbulence in GMCs, so gas at such high densities is largely impervious to external perturbations.

Thus, regardless of the model of star formation one adopts, one would expect that almost all of the gas at densities $\gtrsim 10^{12} \mathrm{~cm}^{-3}$ is part of gravitationally-bound, collapsing objects that have largely decoupled from the 
background turbulent flow. In the absence of effective internal support or external disturbance, order unity of the gas at such high densities is likely to be incorporated into a star within one free-fall time. For this reason, essentially all models of star formation predict that the total mass of gas at densities $\gtrsim 10^{12} \mathrm{~cm}^{-3}$, divided by the free-fall time of this gas, should yield a value comparable to the total star formation rate in the region over which the mass is measured. Instead of $\mathrm{SFR}_{\mathrm{ff}} \sim 0.01$ as for GMCs, i.e. slow star formation, one would obtain $\mathrm{SFR}_{\mathrm{ff}} \sim 1$, i.e. rapid star formation.

However, different models make different predictions about the shape of the curve of $\mathrm{SFR}_{\mathrm{ff}}$ versus density in between $\sim 1 \%$ at the characteristic density of GMCs, $\sim 100 \mathrm{~cm}^{-3}$, and $\sim 1$ at a density $\gtrsim 10^{12} \mathrm{~cm}^{-3}$. These different predictions correspond to different models of the physical scale at which gas both decouples from the background flow and ceases to be supported by internal feedback mechanisms, and thus transitions from slow to rapid star formation. At one extreme, magnetic regulation models such as those of Desch \& Mouschovias (2001), neglecting for the moment turbulent enhancement of the ambipolar diffusion rate (e.g. Heitsch et al. 2004), predict that star formation only becomes rapid once gas decouples from the magnetic field, a process that does not even begin until densities of $\sim 3 \times 10^{10} \mathrm{~cm}^{-3}$. At the other extreme, Bonnell et al. (2003) argue that star clusters form from gas clumps at densities of $\sim 5 \times 10^{4}$ $\mathrm{cm}^{-3}$ that undergo a free-fall collapse in which at least $\sim 30 \%$ of their mass is converted into stars (Kroupa et al. 2001), so star formation should be rapid at this density or higher. In this model, the decoupling scale corresponds to the transition from globally unbound structures (GMCs) to globally bound structures (protoclusters). Thus, extending the Zuckerman \& Evans (1974) calculation of star formation rate divided by free-fall time to higher densities, in hopes of identifying a scale at which there is a transition from slow to rapid star formation, provides a means of distinguishing between models of how star formation is regulated.

It is important at this point to differentiate the concepts of the rate and efficiency of star formation, and the lifetime of star-forming clouds. Unfortunately these terms are often confused in the literature, and there are no standard definitions, so we describe here the definitions we use in this paper. The star formation rate $\dot{M}_{*}$ is the easiest to define, since it is simply the instantaneous conversion rate of gas into stars within some volume. If we pick a density threshold $\rho$, we can then define the dimensionless star formation rate per free-fall time for the gas above that density threshold, $\mathrm{SFR}_{\mathrm{ff}}=\dot{M}_{*} /\left[M(>\rho) / t_{\mathrm{ff}}(\rho)\right]$, where $M(>\rho)$ is the mass of material inside the volume of density $\rho$ or greater. In contrast, the lifetime $t_{\mathrm{cl}}(\rho)$ of star-forming cloud is somewhat more ambiguous. We take it to mean the total duration during which a cloud is visible in a tracer sensitive to densities of $\rho$ or more. Note that our definition neglects the complication that something that starts as a single cloud may in the course of its evolution break up into multiple pieces, so the visible lifetime and the dynamical lifetime may be different. Finally, by the efficiency $\epsilon(\rho)$ we mean the fraction of gas mass $M(>\rho)$ that is converted into stars by a cloud over its lifetime $t_{\mathrm{cl}}(\rho)$.
Again, we neglect the complication that clouds are not closed boxes, so $M(>\rho)$ is likely to be time-dependent due to continuing accretion or mass loss. Ideally $\epsilon(\rho)$ should be computed using a Lagrangian definition of the cloud mass, i.e. all the mass that reached density $\rho$ or more at some point. However, this is generally not a direct observable.

Roughly speaking, the rate, efficiency, and lifetime are related by $\dot{M}_{*} \approx \epsilon(\rho) M(>\rho) / t_{\text {cloud }}(\rho)$. Consequently, one can define a rough time-averaged value for $\mathrm{SFR}_{\mathrm{ff}}$ in a cloud $\left\langle\operatorname{SFR}_{\mathrm{ff}}(\rho)\right\rangle \approx \epsilon(\rho) /\left[t_{\mathrm{cl}}(\rho) / t_{\mathrm{ff}}(\rho)\right]$, i.e. the mass fraction converted into stars divided by the cloud lifetime in free-fall times. One could also describe $\left\langle\mathrm{SFR}_{\mathrm{ff}}\right\rangle$ as being the efficiency per free-fall time, since it measures a fraction of mass converted into stars. However, we refer to it as a rate because it is measured in amount per unit time, whereas efficiency in the literature most commonly refers to the total fraction of mass converted into stars, not the amount per unit time. Moreover, since the time-averaged definition is ambiguous anyway due to uncertainties in exactly what is meant by the efficiency and the lifetime, we will generally use the instantaneous value of $\mathrm{SFR}_{\mathrm{ff}}$, which is well defined and, as we show, directly observable.

The reason for making all these definitions explicit it to point out that observational constraints on $\mathrm{SFR}_{\mathrm{ff}}$ by themselves do not directly constraint $t_{\mathrm{cl}}$ or $\epsilon$, and vice versa. Numerous authors have used various observational techniques to estimate $t_{\mathrm{cl}}$ in clusters and GMCs (e.g. Elmegreen 2000; Hartmann et al. 2001; Tassis \& Mouschovias 2004; Mouschovias et al. 2005; Tan et al. 2006; Ballesteros-Paredes \& Hartmann 2006; Blitz et al. 2005). However, with the exception of $\S 3.5$ we will not discuss the question of cloud lifetime at all in this paper. When we refer to rapid versus slow star formation, what we mean is $\mathrm{SFR}_{\mathrm{ff}} \sim 1$ or $\ll 1$, not is $t_{\mathrm{cl}} \sim t_{\mathrm{ff}}$ or $\gg t_{\mathrm{ff}}$. These questions are conceptually distinct. As an example, note that in the Goldreich \& Kwan (1974) picture of GMCs as collapsing spheres, the evolution time scale is always the cloud free-fall time, $t_{\mathrm{cl}} \sim$ $t_{\mathrm{ff}}$. However, the model still gives $\mathrm{SFR}_{\mathrm{ff}}=1$, and we would therefore describe it as a rapid star formation. In contrast, Ballesteros-Paredes \& Hartmann (2006) argue that star formation lasts only one crossing time of a molecular cloud, so again $t_{\mathrm{cl}} \sim t_{\mathrm{ff}}$, but that during this time only $\sim 1 \%$ of the mass turns into stars. We would describe this as slow star formation, since $\mathrm{SFR}_{\mathrm{ff}} \sim 0.01$, even though the cloud evolution time is similar to that in the Goldreich \& Kwan model. The Zuckerman \& Evans argument rules out the Goldreich \& Kwan free-fall collapse model for GMCs, but is consistent with the Ballesteros-Paredes \& Hartmann model. Thus, we emphasize that in this paper we remain completely agnostic on the question of molecular cloud lifetime.

In this paper we consider star formation in several classes of object. Infrared dark clouds (IRDCs) are regions of high extinction seen in absorption against the Galactic infrared background (Egan et al. 1998; Carev et al. 2000; Simon et al. 2006). IRDCs are clearly associated with star formation, and in at least some cases IRDCs have massive protostars embedded within them (Rathborne et al. 2005). Several authors have suggested (e.g. Menten et al. 2005; Tan 2005; Rathborne et al. 
2006) that IRDCs are the progenitors of star clusters. Within IRDCs, at still higher densities, are dense molecular clumps. These objects may be observed in a variety of molecular transitions with high critical densities, and we consider two here: HCN(1-0) (Gao \& Solomon 2004a b; $\mathrm{Wu}$ et al. 2005) and CS(5-4) (Plume et al. 1997; Shirlev et al. 2003). Molecular clumps seen in these two transitions are often associated with water masers and other signs of massive, clustered star formation. Our goal is to determine $\mathrm{SFR}_{\mathrm{ff}}$ for each of these increasingly dense gas tracers. We also determine this quantity for the Orion Nebula Cluster using a completely different method, which provides an independent check on our estimates.

The remainder of this paper proceeds as follows: in $\S 2$ we use a variety of observations to derive $\mathrm{SFR}_{\mathrm{ff}}$ for our objects and construct a plot of $\mathrm{SFR}_{\mathrm{ff}}$ versus characteristic density to search for signs of a transition from slow to fast star formation. In $\S$, we compare our results to theoretical models for the regulation of star formation, and also point out some implications for the process of star cluster formation. Finally, in $\S$ 政e summarize our conclusions, and suggest directions for future work.

\section{ESTIMATES OF SFR}

Let $\dot{M}_{*}$ be the total star formation rate in a galaxy, and consider star formation occuring in objects of class $\mathrm{X}$. Let $f_{\mathrm{X}}$ be the fraction of galactic star formation that occurs in these objects, $M_{\mathrm{X}}$ be their total mass in the galaxy, and $t_{\mathrm{ff}-\mathrm{x}}$ be their typical free-fall time. Then

$$
\mathrm{SFR}_{\mathrm{ff}-\mathrm{X}}=\frac{f_{\mathrm{X}} \dot{M}_{*} t_{\mathrm{ff}-\mathrm{X}}}{M_{\mathrm{X}}} .
$$

In this section we use observations to estimate all the factors on the right hand side, and thus determine the dimensionless star formation rate $\mathrm{SFR}_{\mathrm{ff}-\mathrm{X}}$ in several classes of object. We emphasize that, as we vary the typical density of the objects we consider, both $t_{\mathrm{ff}-\mathrm{X}}$ and $M_{\mathrm{X}}$ will vary - as we go to denser and denser clouds, the free-fall time will decline, pushing $\mathrm{SFR}_{\mathrm{ff}-\mathrm{x}}$ downward, but the amount of mass in objects of that density will also decrease, pushing $\mathrm{SFR}_{\mathrm{ff}}$ back up. Ultimately, we wish to determine which of these two effects dominate, or whether they roughly balance.

In addition to determining a best value for $\mathrm{SFR}_{\mathrm{ff}-\mathrm{X}}$, we also attempt to make a rough estimate our uncertainties by estimating the uncertainty on each parameter in equation (11). We do this from data where possible and from more general considerations where not, and we add the uncertainty factors in quadrature. This method of combining errors is formally appropriate only if the errors are independent and Gaussian-distributed, neither of which are likely to be completely true, but the procedure does give us a sense of by how much our estimates could vary.

\subsection{Star Formation Rates}

The unknown on the right hand side of (1) that has been studied most heavily is the star formation rate. Numerous authors have discussed methods of inferring the star formation rate from various observables (e.g. Kennicutt 1998a; Iglesias-Páramo et al. 2006, and references therein), so here we only summarize aspects of this discussion that we will apply directly in what follows.

\subsubsection{The Milky Way}

In the Milky Way, McKee \& Williams (1997) find $\dot{M}_{*} \approx 3 M_{\odot} \mathrm{yr}^{-1}$ based on catalogs of Galactic HII regions. Star formation is distributed in an exponential disk with scale radius $H_{R}=3.3 \mathrm{kpc}$ and sharp cutoffs at $3 \mathrm{kpc}$ and $11 \mathrm{kpc}$ in Galactocentric radius. For this distribution, approximately $2 / 3$ of Galactic star formation occurs within the solar circle. The dominant uncertainty in this estimate, roughly 0.3 dex (Kennicutt 1998a), is the shape of the stellar initial mass function (IMF), since HII regions only trace the massive stellar population and one must extrapolate to estimate the total mass. Estimates of the star formation rate based on chemical modelling (Prantzos \& Aubert 1995) also give star formation rates consistent with $3 M_{\odot} \mathrm{yr}^{-1}$ to within a factor of 2 .

\subsubsection{Extragalactic Far-Infrared Observations}

For external galaxies, a commonly-used tracer of star formation is far-infrared (FIR) light. While FIR is usually not the preferred tracer of star formation in normal spiral galaxies like the Milky Way, it is detectable over a very wide range of sources, from ultraluminous infrared galaxies (ULIRGs) to individual clusterforming clumps in the Milky Way, making it possible to look for correlations over a wide range of luminosities (e.g. Gao \& Solomon 2004b; Wu et al. 2005). Kennicutt (1998a) finds that in optically-thick starbursts the IR luminosity and star-formation rate are related by

$$
\dot{M}_{*} /\left(M_{\odot} \mathrm{yr}^{-1}\right)=2 \times 10^{-10}\left(L_{\mathrm{IR}} / L_{\odot}\right) .
$$

The dominant uncertainty is the age of the stellar population, but as we discuss in more detail below, for extragalactic sources this is only $\sim 30 \%$. The uncertainty is considerably larger in normal spiral galaxies, where old stellar populations contribute a significant luminosity, and where only a fraction of the light is reprocessed into infrared. Based on comparisons of multiple tracers of the star formation rate in a range of galaxy types, Iglesias-Páramo et al. (2006) find that (2) probably underestimates the star formation rate in normal spirals. We instead adopt

$$
\dot{M}_{*} /\left(M_{\odot} \mathrm{yr}^{-1}\right)=4 \times 10^{-10}\left(L_{\mathrm{IR}} / L_{\odot}\right)
$$

in normal spirals, which is approximately consistent with the mean in the sample of Iglesias-Páramo et al. (2006) for low star-formation rate $\left(\dot{M}_{*} \lesssim 10 M_{\odot} \mathrm{yr}^{-1}\right)$ systems. There is, however, a much larger scatter in this relation than in the corresponding relation for starburst systems.

\subsubsection{Galactic Far-Infrared Observations}

Several authors (e.g. Plume et al. 1997; Mueller et al. 2002; Shirlev et al. 2003; Wu et al. 2005) use infrared luminosities to estimate the star formation rates within individual cluster-forming gas clumps in the Milky Way. These objects have very high surface densities, so essentially all the light is reprocessed into IR and the uncertainty for spiral galaxies does not apply. However, we will not directly compare to these data because there is a much larger uncertainty arising from the age of the stellar population. In systems $\lesssim 3$ Myr old no stars have disappeared through supernovae, so the massive stellar population cannot yet have reached equilibrium between formation and destruction. 


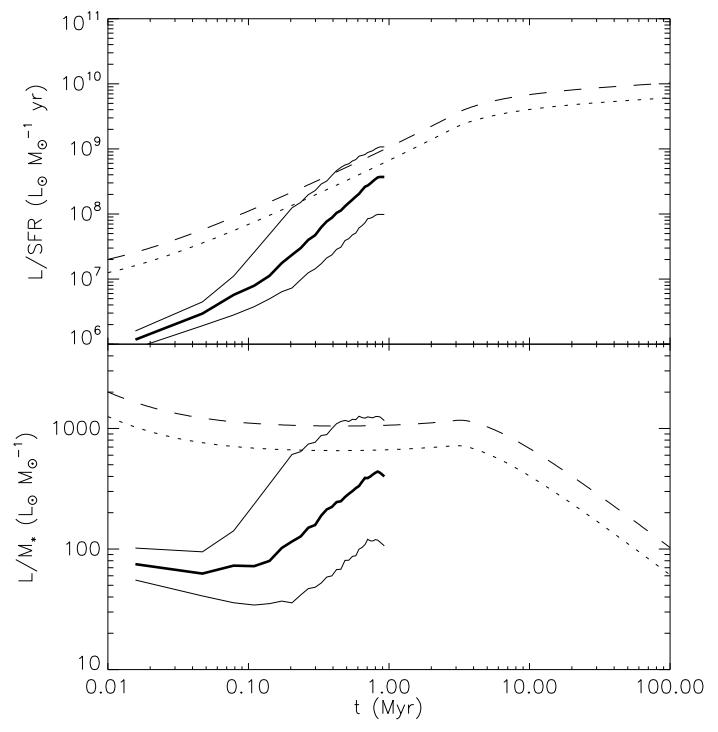

FIG. 1.- Luminosity to star formation rate ratio (upper panel) and luminosity to stellar mass ratio (lower panel) versus time for a stellar population forming at a constant rate. We show the following computations: using starburst99 version 5.0 (Leitherer et al. 1999; Vázquez \& Leitherer 2005) with the default parameters (dashed lines); using starburst99 with an IMF that has a slope of -2.35 from $0.1 M_{\odot}$ to $120 M_{\odot}$ (dotted lines); and the median (thick solid lines) and $1 \sigma$ upper and lower limits (thin solid lines) for 400 clusters simulations computed with the model of Tan \& McKee (2002). Further details are given in $\S 2.1 .3$

To study the magnitude of the uncertainty that this effect induces, we use starburst99 version 5.0 (Leitherer et al. 1999; Vázauez \& Leitherer 2005) to compute the evolution of light to star formation rate and light to mass ratios for systems with constant star formation rates. Figure 11 shows the results. The dashed lines show models using the Kroupa (2002) IMF, while the dotted lines use an IMF with the Salpeter (1955) slope of $\alpha=-2.35$ from $0.1 M_{\odot}$ to $120 M_{\odot}$; for all other starburst99 parameters, we use the defaults. As the plot shows, at ages of a few Myr or more, the luminosity per unit star formation rate is quite insensitive to both age and IMF, so luminosity is a good indicator of star formation rate. However, at younger ages the luminosity in the starburst99 calculation traces total stellar mass more closely than star formation rate, so the light is not a good indicator of star formation rate.

One might think it possible to break this degeneracy by independently determining the age of the stellar population, and then using the luminosity to infer the stellar mass and thus the star formation rate. However, a more detailed treatment of very young systems than starburst99 provides shows that infrared observations alone are not sufficient to constrain the stellar mass in systems $\lesssim 1$ Myr old. In such young systems, a significant fraction of the stars may not yet have contracted to the main sequence, in which case they will be more luminous than equal mass stars on the main sequence would be. Accretion luminosity may further enhance the radiative output above what would be found for a non-accreting population of the same mass. There are also factors that reduce the luminous output compared to an older population of the same mass. Massive stars require $\sim 0.1 \mathrm{Myr}$ to assemble (McKee \& Tan 2002, 2003), so systems younger than this will be missing their contribution to the light. In systems of $\sim 1000 M_{\odot}$ or less, poor sampling of the massive tail of the stellar IMF produces a large scatter, and causes the median system to be less luminous per unit mass or per unit star formation rate than a larger population would be.

We explore how these effects change the light output of a young cluster using the model of Tan \& McKee (2002). In Figure 11 the solid lines show the light output from a simulated cluster with a final stellar mass of $1000 M_{\odot}$ forming at a constant star formation rate of $1.08 \times 10^{-3}$ $M_{\odot} \mathrm{yr}^{-1}$. This model uses the same IMF we use for the Salpeter-slope starburst99 calculation (the dotted line in the figure), but it includes accretion luminosity, pre-main sequence evolution, finite star formation times, and discrete sampling of the IMF. It does not include any postmain sequence evolution. The thick central line shows the median of 400 runs with different samplings of the IMF, and the thin lines above and below it show the tracks that bound $68 \%$ of the runs. As the plots show, the median cluster $\lesssim 1$ Myr in age will be much less luminous per unit star formation rate than a galactic stellar population that is $\gtrsim 10 \mathrm{Myr}$ old and contains enough stars to fully sample the IMF, but the spread can be an order of magnitude for a $1000 M_{\odot}$ cluster. As the cluster mass increases the effects of discrete sampling decrease, causing the luminosity spread to decrease and the median to rise. Even for clusters massive enough to sample the full IMF, though, neither the light to mass ratio nor the light to star formation rate ratio stay constant at ages $\lesssim 1$ Myr, so light is a poor indicator of either stellar mass or star formation rate.

While we cannot use infrared luminosity to study the star formation rate in Galactic star-forming gas clumps directly, there is an observational correlation between infrared and molecular luminosity for such objects (Wu et al. 2005) from which we can learn a great deal. We discuss how to interpret this correlation in light of our results in $\S 3.5$

Finally, note that there are few star clusters where one can estimate the star formation rate directly by placing a large number of stars on the HR diagram and using pre-main sequence tracks to estimate the cluster mass and age spread (e.g. Palla \& Stahler 1999, 2000; Huff \& Stahler 2006). Since this requires luminosity and temperature determinations for many stars, it is posssible only in systems without too much extinction, which limits this technique to low density regions (e.g. Taurus) or somewhat older regions where most of the initial gas is gone (e.g. the Orion Nebula Cluster, ONC). We discuss the ONC in more detail in $\oint$ 2.5 and also refer readers to Tan et al. (2006) for a detailed discussion of other techniques by which one may estimate the formation times of star clusters.

\subsection{Star Formation in Infrared Dark Clouds}

As discussed above, IRDCs are likely to be the progenitors of star clusters. Simon et al. (2006) catalog the infrared dark clouds in the inner Galaxy. This enables us to compute the star formation rate per free fall time in IRDCs by comparing to the total star formation rate 
in the inner Galaxy, roughly $2 M_{\odot} \mathrm{yr}^{-1}$ with a factor of 2 uncertainty. We should note that our calculation of the star formation rate in IRDCs is entirely different from the one given Rathborne et al. (2006). Their estimate assumes an unknown time scale and efficiency of star formation. The calculation we give here does not rely on any such assumptions.

First we must estimate the fraction of star formation that occurs in IRDCs. Lada \& Lada (2003) find that $70-90 \%$ of Galactic star formation occurs in clusters. If a cloud were visible during the entire star formation process as an IRDC, this would imply $f_{\mathrm{IRDC}} \approx 0.8$. However, clouds that have too many embedded protostars will not be infrared dark, and will therefore cease to be visible as IRDCs. This probably does not occur until most of the stars have formed, though, so we adopt $f_{\mathrm{IRDC}}=0.8$ as a reasonable guess, while acknowledging that it could be a bit smaller. Being slighly more conservative about our uncertainty than Lada \& Lada, we adopt $f_{\mathrm{IRDC}}=0.6-1$ as the plausible range of possibilities, i.e. anywhere from $60 \%$ to $100 \%$ of star formation inside the solar circle takes place in IRDCs, corresponding to a factor of 1.25 uncertainty on our estimate of $f_{\text {IRDC }}=0.8$. Note that $f_{\text {IRDC }}=1$ gives the largest possible value of $\mathrm{SFR}_{\mathrm{ff}}$ for IRDCs, so any uncertainties that move $f_{\text {IRDC }}$ out of our plausible range can only make $\mathrm{SFR}_{\mathrm{ff}}$ smaller, not larger.

Rathborne et al. (2006) find that the total mass of IRDCs in the inner Galaxy is $M_{\mathrm{IRDC}} \approx 10^{8} M_{\odot}$ based on the measured properties of a sub-sample of IRDCs observed in $1.2 \mathrm{~mm}$ continuum emission, and an estimate of the detection efficiency for the MSX IRDC survey (Simon et al. 2006). This mass estimate is probably uncertain by a factor of several, because it is unclear how representative the clouds surveyed by Rathborne et al. (2006) are of the entire IRDC population. The sample consists of the darkest clouds (darkness measures a combination of column density and degree of background illumination) from a sample with known kinematic distances. This selection introduces an unknown bias in the mass estimate, so we consider the IRDC mass estimate to be uncertain by factors of a few.

The free-fall time in IRDCs is $t_{\mathrm{ff}}=[3 \pi /(32 G \rho)]^{1 / 2}$, where $\rho$ is the mean density. For the 38 IRDCs in the sample of Rathborne et al. (2006), we define an effective radius $r=(A / \pi)^{1 / 2} D$, where $A$ is the angular area within the $2 \sigma$ detection threshold of the cloud taken from the catalog of Simon et al. (2006), and $D$ is the distance estimate taken from Rathborne et al. We take the mean density to be $\rho=3 M /\left(4 \pi r^{3}\right)$. This procedure is fairly uncertain, since the location of the $2 \sigma$ contour depends on the background emission, and the morphology is filamentary rather than round for a significant minority of clouds. Nonetheless, we can make a rough estimate for $\rho$ and $t_{\mathrm{ff}}$, and check for any systematic variations with IRDC size. We plot the derived free-fall times and densities in Figure 2 As the plot shows, there is a spread of a factor of $\sim 3$, but no clear systematic trend. We adopt as our characteristic free-fall time the mass-weighted harmonic mean

$$
t_{\mathrm{ff}-\mathrm{IRDC}} \equiv \frac{\langle M\rangle}{\left\langle M / t_{\mathrm{ff}}\right\rangle}=0.9 \mathrm{Myr},
$$

corresponding to a number density of hydrogen nuclei

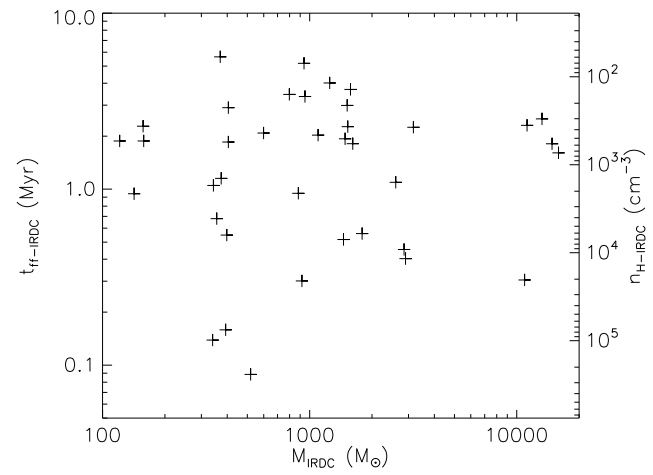

Fig. 2.- Free-fall time and density versus mass for the 38 IRDCs in the sample of Rathborne et al. (2006).

$n_{H}=2 \times 10^{3} \mathrm{~cm}^{-3}$. The range of free-fall times covering the central $67 \%$ of the IRDCs is $0.45-3.0 \mathrm{Myr}$, so the uncertainty is roughly a factor of 2.6. Similarly, the central $67 \%$ of IRDC densities covers a range of $210-9200$ $\mathrm{cm}^{-3}$, an uncertainty of a factor of 6.6.

We now have all the necessary figures to plug into equation (11): the fraction of star formation inside the solar circle taking place in IRDCs is $f_{\mathrm{IRDC}}=0.8$, with a $25 \%$ uncertainty; the total star formation rate inside the solar circle is $2 M_{\odot} \mathrm{yr}^{-1}$, with a factor of 2 uncertainty; the free-fall time in IRDCs is $0.9 \mathrm{Myr}$, with a factor of 4 uncertainty; the total mass of IRDCs in the inner Galaxy is $10^{8} M_{\odot}$, with a hard-to-quantify uncertainty, which we take to be a factor of 3 . Combining these estimates gives a fiducial value $\mathrm{SFR}_{\mathrm{ff}-\mathrm{IRDC}} \approx 0.014$. Combining the errors as described in $\S 2$ gives an uncertainty of a factor of 4.6.

\subsection{Star Formation in Gas Traced by $H C N$}

The molecular transition $\mathrm{HCN}(1-0)$ has a critical density of $n_{H}=6 \times 10^{4} / \tau \mathrm{cm}^{-3}$ (c.f. Gao \& Solomon 2004a, who give the critical density in terms of number density of hydrogen molecules rather than hydrogen nuclei), where $\tau$ is the line-center optical depth in the escape probability approximation, and is therefore an excellent tracer of the dense molecular regions associated with star formation. Gao \& Solomon (2004a b) observe a large sample of normal spiral galaxies and luminous and ultraluminous infrared galaxies in $\mathrm{HCN}(1-0)$ and show that the HCN luminosity correlates well with the IR luminosity, following

$$
\frac{L_{\mathrm{IR}}}{L_{\odot}}=911 \pm 227 \frac{L_{\mathrm{HCN}}}{\mathrm{K} \mathrm{km} \mathrm{s}^{-1} \mathrm{pc}^{-2}},
$$

where the figure quoted is the mean plus or minus the standard deviation. Galactic cores with luminosities above $10^{4.5} L_{\odot}$ observed in $\mathrm{HCN}$ by Wu et al. (2005) show a similar correlation, with almost exactly the same mean, although the median is lower than the median of the extragalactic sample by a factor of 2.5. Since infrared luminosity is a tracer of star formation rate, and $\mathrm{HCN}(1-0)$ is a tracer of molecular gas, this relation is a direct measure of the star formation rate per unit mass in gas of densities traced by HCN, and thus of $\mathrm{SFR}_{\mathrm{ff}}$ in that gas. We use the difference in medians between the Galactic and extragalactic samples as an estimate of the 
uncertainty factor in the IR-HCN correlation, although we note that this is almost certainly an overestimate for the extragalactic sample because that data set is much more tightly correlated than the Galactic sample.

To derive the total gas mass that corresponds to a given luminosity $L_{\mathrm{HCN}}$ in the $\mathrm{HCN}(1-0)$ line, Wu et al. (2005) observe in $\mathrm{HCN}(1-0)$ a sample of star-forming clumps with known virial masses determined from optically-thin $\mathrm{C}^{34} \mathrm{~S}$ emission. (Other methods of estimating the mass of these objects that do not assume virial balance, and make other assumptions about the emission, e.g. Plume et al. 1997, Mueller et al. 2002, Shirlev et al. 2003, give masses that are comparable to or better than the uncertainty in the mass estimate we adopt below.) They find a median ratio $M_{\mathrm{HCN}} / M_{\odot}=6 L_{\mathrm{HCN}} /\left(\mathrm{K} \mathrm{km} \mathrm{s}^{-1} \mathrm{pc}^{2}\right)$, and a mean of 11. Gao \& Solomon (2004a) perform radiative transfer calculations under a variety of assumptions about the temperature and distribution of the $\mathrm{HCN}$ emitting gas, and arrive at conversion factors of $10-25$. For normal spiral galaxies, we adopt a conversion of $M_{\mathrm{HCN}} / M_{\odot}=11 L_{\mathrm{HCN}} /\left(\mathrm{K} \mathrm{km} \mathrm{s}^{-1} \mathrm{pc}^{2}\right)$, the mean value measured by Wu et al. (2005). To be conservative we estimate that the conversion is uncertain by a factor of 3 , corresponding to a range that is larger than the range of $6-25$ spanned by the various estimates.

Recent $\mathrm{HCO}^{+}$observations suggest that chemical changes triggered by X-rays may enhance the HCN abundance in galaxies with an AGN (Graciá-Carpio et al. 2006). This reduces the mass estimate for a given HCN luminosity by a factor of $\sim 2$ in starbursts, most of which have at least a small AGN. For starbursts we therefore modify our light to mass conversion to $M_{\mathrm{HCN}} / M_{\odot}=$ $5.5 L_{\mathrm{HCN}} /\left(\mathrm{K} \mathrm{km} \mathrm{s}^{-1} \mathrm{pc}^{2}\right)$, again with roughly a factor of 3 uncertainty.

Combining this with the conversion from IR luminosity to star formation rate discussed in $\S 2.1 .2$ for both starburst galaxies and normal spirals gives $\dot{M}_{*} / M_{\mathrm{HCN}} \approx$ $(30 \mathrm{Myr})^{-1}$. Adding the uncertainties of a factor of 2.5 on the IR-HCN correlation, 3 on the HCN to mass conversion, and 1.3 on the IR to star formation rate conversion gives a combined uncertainty of a factor of 4.1.

The free-fall time depends on the mean density, for which a rough guess based on the optical thickness of the HCN emission is $n_{H} \sim 6 \times 10^{4} \mathrm{~cm}^{-3}$. The true density of the HCN-emitting gas could be lower or higher depending on optical depth and beam-filling effects, as appears to be the case with $\mathrm{CS}(5-4)$ emission (see the discussion below in $\S$ 2.4). However, this is unlikely because the mass to luminosity ratio for $\mathrm{HCN}(1-0)$ inferred from radiative transfer calculations is very close to that inferred from correlation of HCN luminosity with virial masses for objects seen in the Galaxy. This suggests that the HCN emission for Galactic HCN clumps is close to beam-filling and is not extremely self-absorbed, so the density of the objects being observed is unlikely to be very different than $n_{H} \sim 6 \times 10^{4} \mathrm{~cm}^{-3}$. If anything, the observations of $\mathrm{Wu}$ et al. (2005) suggest that the mean density may be slightly larger than $n_{H} \approx 6 \times 10^{4} \mathrm{~cm}^{-3}$ (also J. Wu, 2006, private communication). We adopt a mean density of $n_{H} \sim 6 \times 10^{4} \mathrm{~cm}^{-3}$ as a fiducial value, giving $t_{\mathrm{ff}-\mathrm{HCN}} \approx 0.18 \mathrm{Myr}$, and estimate that the uncertainty is no more than a factor of 2 , corresponding to a factor of 4 change in the density.
Finally, since the extra-Galactic observations include all $\mathrm{HCN}(1-0)$ emission from the target galaxy, and all star-formation occurs at densities high enough to be traced by $\mathrm{HCN}$ emission, we set $f_{\mathrm{HCN}}=1$.

Using our fiducial estimates for $\dot{M}_{*} / M_{\mathrm{HCN}}, f_{\mathrm{HCN}}$, and $t_{\mathrm{ff}-\mathrm{HCN}}$ in (11) gives $\mathrm{SFR}_{\mathrm{ff}-\mathrm{HCN}}=0.0058$. The factor of 4.1 uncertainty in our value of $\dot{M}_{*} / M_{\mathrm{HCN}}$, combined with the factor of 2 uncertainty in our free-fall time, gives a complete uncertainty of a factor of 4.6.

\subsection{Star Formation in Gas Traced by $C S$}

The CS(5-4) line has a critical density of $n_{H} \approx 1.5 \times$ $10^{6} / \tau \mathrm{cm}^{-3}$, and thus traces gas at even higher densities than HCN(1-0). Plume et al. (1997) and Shirlev et al. (2003) survey in CS emission lines a sample of regions selected from sources reported in the Arcetri $\mathrm{H}_{2} \mathrm{O}$ maser catalog (Valdettaro et al. 2001) that are thought based on IRAS colors to be associated with star formation. From the mean CS(5-4) luminosity of their targets and the sky coverage fraction of the maser catalog, they estimate that the total CS(5-4) luminosity of the Galaxy is at least $L_{\mathrm{CS}} \approx 20 L_{\odot}$. This is a lower limit only, because the maser catalog may not be complete over the region of sky it covers, there is probably at least some Galactic CS(5-4) emission that is not correlated with water masers, and Shirlev et al. (2003) only detect $75 \%$ of their targets in $\mathrm{CS}(5-4)$, while they detect $90 \%$ in all the transitions for which they look. Nonetheless, this lower limit on luminosity gives a lower limit on the amount of gas in the Galaxy that is sufficiently dense to produce CS(5-4) emission, which we can in turn use in equation (11) to obtain an upper limit on $\mathrm{SFR}_{\mathrm{ff}}$ at $\mathrm{CS}$ densities.

First we must estimate the CS(5-4) "X" factor to convert the luminosity to a mass. Like $\operatorname{HCN}(1-0)$, starforming clumps are usually optically thick in CS(5-4), so it is reasonable to expect that such an $\mathrm{X}$ factor might exist. Figure 3 shows the virial mass $M_{\text {vir }}$ (determined from optically-thin $\mathrm{C}^{34} \mathrm{~S}$ emission) versus $L_{\mathrm{CS}}$ for the $57 \mathrm{ob}-$ jects in the Shirlev et al. (2003) sample. The correlation is well fit by the line $M_{\mathrm{CS}} / M_{\odot}=4.5 \times 10^{4} L_{\mathrm{CS}} / L_{\odot}$ over more than two orders of magnitude in mass. This implies a total Galactic mass in CS clumps of $M_{\mathrm{CS}} \geq 9 \times 10^{5}$ $M_{\odot}$. The uncertainty of the fit, done in the logarithm of the data, is a factor of 3.3, and we adopt this as the uncertainty in our luminosity to mass correlation. However, as we discuss more below, the virial mass gives the lowest mass of the three estimators tested by Plume et al. (1997), by roughly a factor of 3 , so the true mass is much more likely to be larger than our value rather than smaller.

Note that this mass estimate strongly suggests that the true CS mass in the Milky Way is substantially larger. CS and HCN emission often come from overlapping regions in the Milky Way (Wu et al. 2005), and in such overlapping regions the area emitting CS is not vastly smaller than the area emitting HCN. However, the total mass of CS gas that our estimate suggests is smaller than the mass of HCN-emitting gas that Gao \& Solomon $(2004 \mathrm{a}$ b) see in galaxies like the Milky Way by $1-2$ orders of magnitude. The most likely explanation is incompleteness of the CS survey.

To estimate the free-fall time, we must know the gas density. Plume et al. (1997), based on radiative trans- 


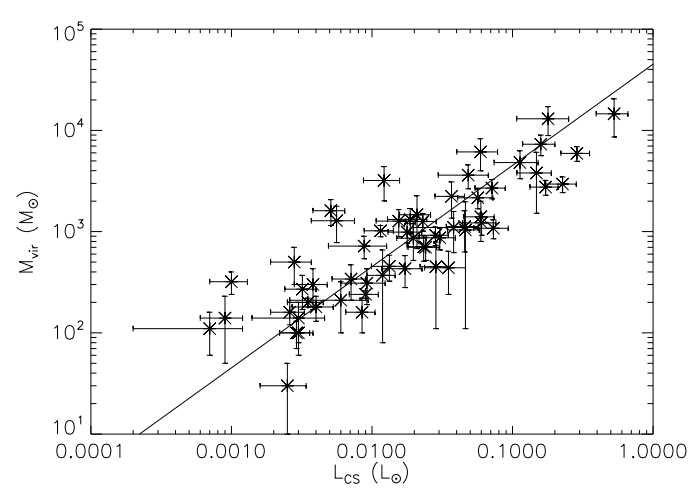

Fig. 3.- Virial mass $M_{\text {vir }}$ versus luminosity in the CS(5-4) line $L_{\mathrm{CS}}$ (asterisks with error bars) for objects in the Shirley et al. (2003) sample. We also show the best-fit linear correlation (line).

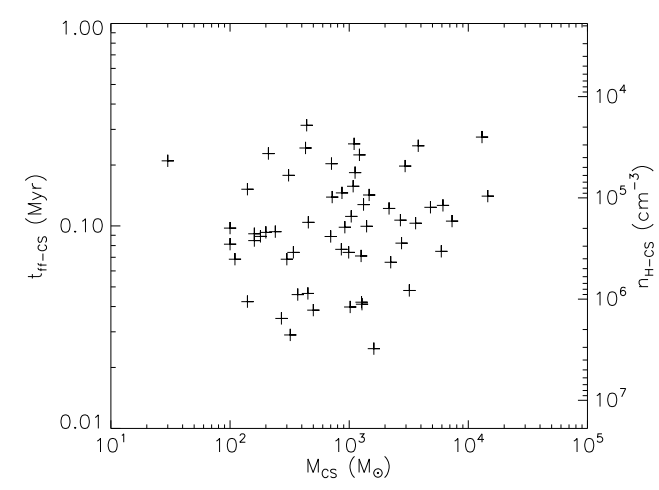

FIG. 4. - Free-fall time and density versus mass for the 57 CS(5-4) clumps in the sample of Shirley et al. (2003).

fer modeling, find a mean density of $n_{H}=1.6 \times 10^{6}$ $\mathrm{cm}^{-3}$, roughly the critical density. However, this average is intensity-weighted, so it may overestimate the true mean. Plume et al. (1997) and Shirlev et al. (2003) find that the virial mass for objects in their survey is systematically smaller than the mass estimated by assuming that all the gas is at the density inferred from the radiative transfer calculations. Based on the difference in mass estimates, they conclude that the filling factor of gas at densities of $n_{H} \sim 2 \times 10^{6} \mathrm{~cm}^{-3}$ or higher is typically $\sim 0.3-0.5$. Thus, the density of $n_{H}=1.6 \times 10^{6}$ $\mathrm{cm}^{-3}$ probably represents an upper limit on the true density. We therefore compute the mean density for the 57 clumps in the Shirley et al. catalog from the virial mass and deconvolved half-peak radius (Shirley et al.'s $R_{\mathrm{CS}}$ ) to compare to the result of the radiative transfer calculations. We show our derived densities and the corresponding free-fall times in Figure 4 The mass-weighted harmonic mean free-fall time for the CS clumps (computed from equation 4) is $t_{\mathrm{ff}-\mathrm{CS}}=0.10 \mathrm{Myr}$, and we adopt this as our fiducial value. The range $0.05-0.2 \mathrm{Myr}$ covers the central $67 \%$ of the free-fall times, so we take the uncertainty to be a factor of 2 . The density corresponding to our adopted mean free-fall time is $n_{H}=1.8 \times 10^{5} \mathrm{~cm}^{-3}$, and the range in free-fall times corresponds to a density range of a factor of 1.4 .

Finally, the estimated CS luminosity comes from sources associated with water masers, which arise only in regions of massive, clustered star formation. This implies a maximum of $f_{\mathrm{CS}}=0.8$. Alternately, we would obtain the same result by assuming that all star-forming regions produce $\mathrm{CS}(5-4)$ emission and raising our estimate of $M_{\mathrm{CS}}$ by a factor of $1 / 0.8$ to account for the fraction of $\mathrm{CS}(5-4)$ emission associated with water masers. In either case, our value for either $f_{\mathrm{CS}}$ or $M_{\mathrm{CS}}$ is an upper limit because we do not know what fraction of clustered star formation is associated with water maser emission. Combining $f_{\mathrm{CS}}, M_{\mathrm{CS}}$, and $t_{\mathrm{ff}-\mathrm{CS}}$ in equation (1) gives $\mathrm{SFR}_{\mathrm{ff}-\mathrm{CS}} \leq 0.27$, with an uncertainty of a factor of 3.6 . Again, it seems likely that the true value is significantly lower than this upper limit.

\subsection{Star Formation in the Orion Nebula Cluster}

We can add one more point for a specific object. The Orion Nebula Cluster (ONC) is the only star cluster that has been studied well enough so that it is possible to make reasonable estimates of the stellar mass, the formation time, and the properties of the progenitor gas system, allowing direct determination of $\mathrm{SFR}_{\mathrm{ff}}$. This is quite useful because it provides an estimate of $\mathrm{SFR}_{\mathrm{ff}}$ that does not depend on conversions from luminosities to masses or star formation rates, and thus is subject to completely different systematic errors than the methods we have used thus far.

The total stellar mass of the ONC is $4600 M_{\odot}$ (Hillenbrand \& Hartmann 1998). Tan et al. (2006) analyze several lines of evidence to conclude that formation of the ONC took place over at least $3 \mathrm{Myr}$, and possibly longer - see Huff \& Stahler (2006). The age estimate comes from a combination of pre-main sequence fitting, which is fairly reliable for stars $\gtrsim 2$ Myr in age, and from the age of a dynamical ejection event. We refer readers to Tan et al. (2006) for the detailed evidence. The free-fall time in the current stellar system is $t_{\mathrm{ff}}=0.5$ Myr, but it must have been smaller in the progenitor gas system, since some of the mass has been expelled by the Trapezium stars, and the cluster may be expanding. Uncertainties in these processes lead to a range of mass estimates for the progenitor, ranging from $\sim 15000$ $M_{\odot}$ if the ONC today is marginally unbound and expanding (Kroupa et al. 2001) to $6700 M_{\odot}$ if the ONC is currently bound and non-expanding (Huff \& Stahler 2006), although the latter would imply an extraordinarily high star formation efficiency. If we neglect the possibility that the cluster has undergone significant expansion since expelling its gas, this implies that the free-fall time in the progenitor gas system was $t_{\mathrm{ff}}=0.3-0.4$ Myr, corresponding to a density $n_{H}=1-2 \times 10^{4} \mathrm{~cm}^{-3}$. It seems unlikely that star formation began when most of the gas in the ONC was spread over a much larger region at lower density, both because there is no correlation between the age and spatial distribution for stars in the ONC (Huff \& Stahler 2006) and because there must have been a system sufficiently dense to lead to dynamical interactions and ejection of massive stars 2.5 Myr ago (Hoogerwerf et al. 2001; Tan et al. 2006).

Combining the initial gas mass, final stellar mass, and initial free-fall time implies that in Orion $\mathrm{SFR}_{\mathrm{ff}}=$ $0.03-0.09$. The true value is likely to be towards the low end of this range, since even if the $\mathrm{ONC}$ is bound it has almost certainly undergone some expansion from its original gaseous state, meaning that the free-fall time we 
have adopted is probably too large.

\subsection{Summary of Observations}

We summarize our results by plotting the dimensionless star formation rate $\mathrm{SFR}_{\mathrm{ff}}$ versus characteristic density in Figure 5. In addition to our points for CS clumps, HCN clumps, IRDCs, and the ONC, we can add a point for GMCs as a whole, in which the typical density and free-fall time are $n=100 \mathrm{~cm}^{-3}$ and $t_{\mathrm{ff}}=4.4$ Myr (McKee 1999). The total mass of GMCs in the Galaxy is roughly $10^{9} M_{\odot}$ (Bronfman et al. 2000), so using the same argument as in $\S 2.2$ gives $\mathrm{SFR}_{\mathrm{ff}} \approx 0.013$. Krumholz \& McKee (2005) give a much more detailed calculation of this value, but for simplicity we adopt rough numbers here. Since the GMC population in the Milky Way is reasonably well characterized, the uncertainty in $\mathrm{SFR}_{\mathrm{ff}}$ is dominated by uncertainty in the star formation rate, so it is about a factor of 2 . The range of densities covered by star-forming GMCs is roughly a factor of 3, so we take this range and the range in $S_{F R}$ to roughly define our uncertainty for GMCs.

\section{IMPLICATIONS AND COMPARISON TO THEORY}

The star formation rate per free-fall time is a key prediction of any model for a physical mechanism that regulates the star formation rate. The most important result summarized by Figure [5] which any successful theory must be able to explain, is that there is no evidence for a transition to rapid star formation out to densities of $n \sim 10^{5} \mathrm{~cm}^{-3}$. Even given the large uncertainties in the data, this conclusion is quite firm. If any of the objects we have analyzed were bound, detatched from the largescale turbulent flow, and collapsing without significant impedance from feedback, we would expect $\mathrm{SFR}_{\mathrm{ff}} \sim 1$, while even the top of the uncertainty range for the data point with the highest value of $\mathrm{SFR}_{\mathrm{ff}}$ is below 0.1 . Only the CS data is consistent with a transition for rapid star formation, and this is an upper limit for which there is strong evidence to suggest that the true value is significantly lower.

A second conclusion, which is much more tentative, is that we do not see any significant change in SFR $_{\mathrm{ff}}$ with density at all. Given the uncertainties, obviously we are insensitive to changes in $\mathrm{SFR}_{\mathrm{ff}}$ smaller than roughly an order of magnitude, and the somewhat higher point for the ONC suggests that it is possible there is some modest rise in $\mathrm{SFR}_{\mathrm{ff}}$ in star clusters. Any such rise with density, though, must be fairly small over the range of density we have explored. In summary, the data are consistent with $\mathrm{SFR}_{\mathrm{ff}}$ of a few percent regardless of density, and require $\mathrm{SFR}_{\mathrm{ff}} \lesssim 0.1$ to densities of at least $6 \times 10^{4} \mathrm{~cm}^{-3}$. The characteristic scale at which one transitions from slow to fast star formation must lie at even higher densities. Here we investigate how well various models of star formation explain this result, and discuss a few of its implications.

\subsection{Unbound GMC / Collapsing Cluster Models}

One proposed explanation for the low star formation rate in molecular clouds is that GMCs are not bound by gravity (Mac Low \& Klessen 2004; Clark \& Bonnell 2004; Clark et al. 2005; Dobbs et al. 2006). In this model, GMCs are transient over-densities created by turbulence or spiral shocks in the atomic ISM. Since they are confined by ram pressure, they re-expand and disperse in roughly one dynamical time. The star formation rate appears low because only a small fraction of the mass is gravitationally bound and can collapse, but that mass which can collapse will form stars rapidly. Feedback is not effective at slowing collapse or establishing an equilibrium, and only stops the star formation process at its end by rapidly unbinding the cluster (Bonnell \& Bate 2006). Star formation in protoclusters is rapid but is halted after a short period by feedback. Thus, the star formation rate is high, even if the duration is short, and this should be detectable as a rise in $\mathrm{SFR}_{\mathrm{ff}}$ at the typical density of protoclusters.

Simulations based on these premises allow us to compare these models to the observational data. Before discussing individual simulations, we note that we cannot analyze the simulation data as thoroughly as we have analyzed the observational data. Ideally, one would take a snapshot of a simulation at various points in time, compute the mass $M(>\rho)$ that is denser than $\rho$, make a plot of $M(>\rho) / t_{\mathrm{ff}}(\rho)$, and compare that to the instantaneous star formation rate in the simulation. This is effectively what we have done with the observational data. However, simulation papers generally do not report $M(>\rho)$. They only give a single density, usually the initial (uniform) density, and report a total mass of stars formed over the course of the entire simulation. Thus we perform our calculations using $M(>\rho)$ for the initial density at the initial time, and compare the mean star formation rate over the entire simulation. This should give a rough average value that can stand in for a more detailed calculation of $M(>\rho) / t_{\mathrm{ff}}(\rho)$ as a function of density and time. However, we encourage authors of future simulations of GMC evolution or star cluster formation to compute $M(>\rho) / t_{\mathrm{ff}}(\rho)$ for at least a few time slices to facilitate comparison to observations.

Clark et al. (2005) estimate from their simulations that unbound GMCs would convert $5-10 \%$ of their mass into stars in $2-3$ free-fall times, giving $\mathrm{SFR}_{\mathrm{ff}} \sim 0.03$. In contrast, Bonnell et al. (2003) simulate a marginally-bound $1000 M_{\odot}$ clump with an initial radius of $0.5 \mathrm{pc}$, giving $n_{H}=5.5 \times 10^{4} \mathrm{~cm}^{-3}$, roughly the properties of an HCN clump. There is no feedback, and the turbulence decays freely. They find that, after 2.6 initial free-fall times, $58 \%$ of the mass has gone into stars, giving $\mathrm{SFR}_{\mathrm{ff}}=0.22$. This is probably an underestimate of the true value of $\mathrm{SFR}_{\mathrm{ff}}$ in this scenario, since the simulation starts with a uniform density and thus star formation does not really begin for roughly 1 free-fall time. If we instead measured from the time when the first star formed to the time when the last one formed, we would find an even larger value of $\mathrm{SFR}_{\mathrm{ff}}$.

This is consistent with other simulations in which the turbulence is weak. For example, Klessen \& Burkert (2000) model formation of a star cluster by simulating a periodic box in which the gravitational potential energy greatly exceeds the kinetic energy, and find that their simulation converts $60 \%$ of the initial mass into stars in 1.8 free-fall times, giving $\mathrm{SFR}_{\mathrm{ff}}=0.33$. The simulation is scale-free, but Klessen \& Burkert suggest that the model should be reasonable for a region with a typical density of $n_{H} \sim 10^{5} \mathrm{~cm}^{-3}$. Vázquez-Semadeni et al. (2003) perform a parameter study of regions with turbulent driving of various strengths and find that when the turbulent driving is weak so that the system is unstable to global 


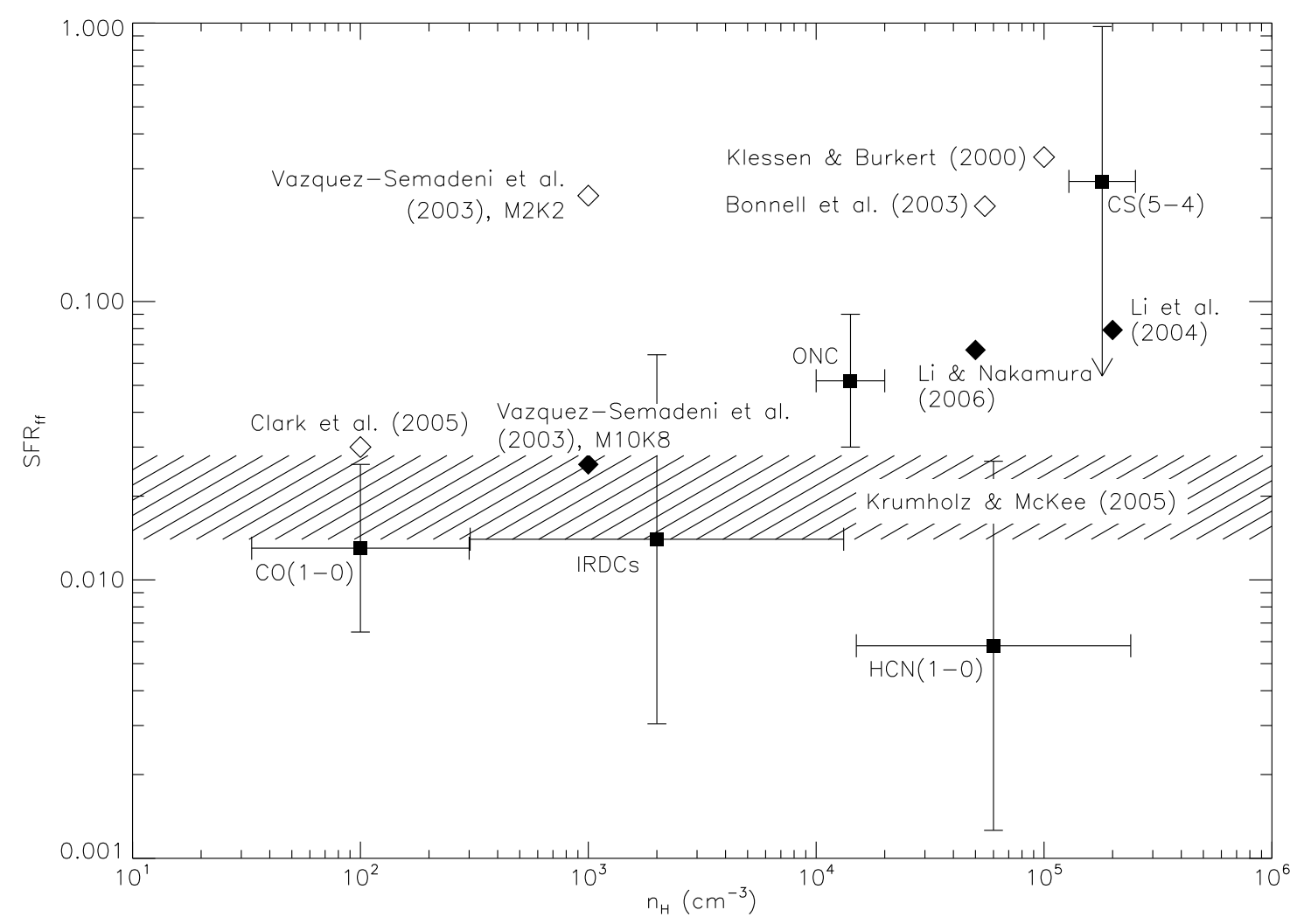

FIG. 5.- Estimates of the dimensionless star formation rate $\mathrm{SFR}_{\mathrm{ff}}$ in objects of characteristic density $n_{H}$. We show observational estimates (filled squares with error bars), an observational upper limit (filled square with arrow), simulations with weak or decaying turbulence (open diamonds), simulations with strong, driven turbulence (filled diamonds), and the prediction of an analytic model (hatched region). For the observational points, we label each point by the tracer used to estimate that point. We label the simulation points and the analytic model region by the reference for the simulation or model. The simulations of Klessen et al. (2000), Vázquez-Semadeni et al. (2003), and Li et al. (2004) are dimensionless, so the values of $n_{H}$ for them are arbitrary. We have adopted the authors' suggested scale value of $n_{H}$ where one is given, or an arbitrary value otherwise. For the simulations of Vázquez-Semadeni et al., we show a weakly-driven model (M2K2, meaning Mach number 2, driving wavelength of $1 / 2$ the box size), and a strongly-driven one (M10K8), and we derive values of $\mathrm{SFR}_{\mathrm{ff}}$ for these models via the method described in Krumholz \& McKee (2005). The region indicated for the Krumholz \& McKee model corresponds to the parameter range $\alpha_{\mathrm{vir}}=1-2, \mathcal{M}=20-40$.

collapse, $\mathrm{SFR}_{\mathrm{ff}} \sim 0.3$ is a typical value. (As we discuss below in $\$$ 3.4. Vázquez-Semadeni et al. 2003 find that if the turbulent driving is strong, the simulations produce lower values of $\mathrm{SFR}_{\mathrm{ff}}$ that agree much better with the observations.)

We place points from the simulations of Klessen \& Burkert (2000), Bonnell et al. (2003), and Clark et al. (2005), and from one of the weakly-driven turbulence simulations of Vázquez-Semadeni et al. (2003), on Figure 5 This indicates a clear observational problem for these models. They produce roughly the correct star formation rate at the GMC scale, but are inconsistent with the data on the IRDC and HCN scale at the order of magnitude level. They are consistent with the CS data only if the value of $\mathrm{SFR}_{\mathrm{ff}}$ in CS clumps is near its upper limit. We conclude that models where star formation occurs in unbound GMCs but freely collapsing dense clumps, or where the turbulence in those clumps is driven but at very sub-virial levels, are ruled out by the data. Star formation in dense clumps cannot occur through global collapse.

We emphasize again that one cannot avoid this problem by hypothesizing a feedback process that rapidly destroys clumps once they have turned a relatively small fraction of their mass into stars, but retaining the picture of star formation occuring in clumps that are undergoing global collapse. Even if feedback destroyed collapsing clumps, while they existed they would still be forming stars at a rate much larger than $\mathrm{SFR}_{\mathrm{ff}}$ of a few percent, which is inconsistent with the data. Only if destruction by feedback prevents global collapse from starting can it produce the observed value of $\mathrm{SFR}_{\mathrm{ff}}$. In this case, though, estimates that cluster-forming clumps turn $\sim 30 \%$ of their gas into stars (Kroupa et al. 2001; Lada \& Lada 2003) require that global collapse be held off for much longer than a free-fall time. We discuss the implications of this in more detail in $\S 3.5$

One final caveat worth mentioning in our interpretation of these simulations is that once a significant fraction of the particles in an SPH simulation have been accreted by sink particles, the effective resolution of that code, and its ability to follow the hydrodynamics, may be significantly degraded. It is unclear how large this effect is, and whether it would tend to increase or decrease $S_{F}$. However, since the effect is only a problem late in the simulations, it seems unlikely that it could shift $\mathrm{SFR}_{\mathrm{ff}}$ by more than a few percent. 


\subsection{Galactic-Scale Gravitational Instability Models}

The criticism of unbound GMC models applies in a much weaker form to models in which the star formation rate is determined by large-scale global gravitational instability in a galactic disk (e.g. Li et al. 2005a b, 2006; Tasker \& Brvan 2006). These models postulate that the star formation rate in galaxies is determined by the rate at which gravitational instability in the galactic disk drives gas to high densities, and that the strength of this gravitational instability determines the star formation rate. This is probably at least partly true, in that the star formation rate is set partly by the supply of molecular gas, which is in turn controlled by gravitational instability. However, roughly one third of the mass of the Milky Way's ISM is in molecular clouds. Clearly the rate-limiting step in star formation is not at the stage of converting atomic ISM to molecular gas, but somewhere at higher density. For this reason, the gravitational instability models provide only a partial description of the star formation process.

In practice, this limitation is reflected in the star formation prescriptions that simulations of galactic-scale gravitational instability adopt. These simulations cannot resolve structures of molecular cloud size or smaller. Instead, when such structures form the simulations use a recipe to determine the amount of gas that turns into stars. These prescriptions only reproduce the correct star formation rate if they correspond to rates $\mathrm{SFR}_{\mathrm{ff}} \ll 1$ in the dense gas.

For example, Tasker \& Brvan (2006) assume for their highest resolution simulations that in cells with densities above $10^{3} \mathrm{~cm}^{-3}, 50 \%$ of the gas is converted into stars per free-fall time of that gas. This corresponds to $\mathrm{SFR}_{\mathrm{ff}}=0.5$. However, examining their Figure 8 shows that this leads to a star formation rate that is an order of magnitude larger than the observed Kennicutt (1998b) law without feedback, and a half an order of magnitude too large if they include feedback. They also find that even reducing their value of $\mathrm{SFR}_{\mathrm{ff}}$ by a factor of 10 is not by itself sufficient to reproduce the Kennicutt law. This is not surprising based on our results that $\mathrm{SFR}_{\mathrm{ff}}$ is at most a few percent in gas at densities of $10^{3} \mathrm{~cm}^{-3}$. Reproducing the observed star formation law would require a value for $\mathrm{SFR}_{\mathrm{ff}}$ of this order.

In the simulations of $\mathrm{Li}$ et al. (2005a, b, 2006), the authors are able to reproduce the Kennicutt (1998b) law by adopting a prescription for star formation whereby collapsed mass is assumed to form stars with a 30\% "efficiency", in the sense that they count $30 \%$ of the gas that is accreted onto sink particles in the simulation as stars. The remaining $70 \%$ of the mass stays in the sink particles, but is assumed not to form stars. There is no simple way to convert this recipe into a value of $\mathrm{SFR}_{\mathrm{ff}}$, since it specifies that the star formation rate in any particle is $30 \%$ of the particle's current accretion rate, and we do not have available the full time history of accretion for each particle. Even if we did have this information, interpretation would be diffuclt because the gas assumed not to form stars remains locked up in sink particles indefinitely, rather than being ejected back into the ISM by feedback as happens in real star clusters. This greatly reduces the supply of gas available to form stars. Nonetheless, the fact that these simulations need to adopt a subgrid model that significantly reduces the collapsed mass counted as stars in order to reproduce the Kennicutt law is consistent with our point that one can only explain the observed rates of star formation if there is some mechanism that inhibits star formation even in the densest gas that the simulations can resolve.

The reason that these simulations reproduce the observed star formation rate only if they have $\mathrm{SFR}_{\mathrm{ff}} \ll 1$ is clear in light of Figure [5] if the gas in IRDCs or HCN gas were collapsing on its free-fall time, the star formation rate in the Milky Way would exceed the observed value by two orders of magnitude. To obtain the correct overall star formation rate, one must adopt a star formation rate $\mathrm{SFR}_{\mathrm{ff}} \ll 1$ even in the densest gas that the simulations are currently capable of resolving. While this is an entirely reasonable approach in simulations, a theoretical understanding of the star formation rate requires an explanation why $\mathrm{SFR}_{\mathrm{ff}} \ll 1$ even in gas that is already collapsed to densities above the critical density of $\mathrm{HCN}(1-0)$, five orders of magnitude higher than the mean in the Galactic ISM. This point does not mean that global gravitational instability is unimportant in regulating star formation, simply that it cannot be the sole agent. Thus, models such as these are complementary to models of SFR $\mathrm{ff}_{\text {in }}$ dense gas such as those of Krumholz \& McKee (2005).

\subsection{Magnetic Regulation Models}

Another possible explanation for low star formation rates is strong magnetic fields (e.g. Shu et al. 1987; McKee 1989; Tassis \& Mouschovias 2004; Nakamura \& Li 2005). If star-forming clouds are magnetically subcritical, which is controversial on both observational and theoretical grounds (McKee et al. 1993; Crutcher 1999; Bourke et al. 2001; Padoan et al. 2004; Heiles \& Crutcher 2005), then they cannot collapse before ambipolar diffusion allows the magnetic field to slip out of the gas, and star formation proceeds on the ambipolar diffusion time scale instead of the free-fall time scale. For a uniform gas, the time scale required for ambipolar diffusion to decouple the gas and the field is (Shu 1992)

$$
t_{\mathrm{AD}} \sim \frac{L^{2}}{v_{A}^{2}} \gamma C \rho^{1 / 2},
$$

where $L$ and $\rho$ are the characteristic size scale and density of the cloud, $v_{A}$ is the Alfvén speed, $\gamma \approx 3.5 \times 10^{13}$ $\mathrm{cm}^{3} \mathrm{~g}^{-1} \mathrm{~s}^{-1}$ is the ion-neutral drag coefficient, and $C=$ $3 \times 10^{-16} \mathrm{~cm}^{-3 / 2} \mathrm{~g}^{1 / 2}$ is the cosmic ray ionization constant. (In high column density environments like IRDCs, interstellar UV photons cannot penetrate and ionization is dominated by cosmic rays.) If we assume that this describes the star formation timescale, then the star formation rate should obey roughly (Ciolek \& Mouschovias 1993)

$$
\mathrm{SFR}_{\mathrm{ff}} \sim \frac{\epsilon_{\mathrm{c}} t_{\mathrm{ff}}}{t_{\mathrm{AD}}}
$$

where $\epsilon_{\mathrm{c}} \approx 0.5$ is the fraction of the mass in a prestellar core that reaches the star rather than being ejected by outflows (Matzner \& McKee 2000).

To determine what this implies for $\mathrm{SFR}_{\mathrm{ff}}$, we need to know how the magnetic field behaves in objects of 
varying densities. While there are very few direct observations of magnetic fields for extremely dense objects of the sort we are considering, observations do show that magnetic fields obey the correlation $B=$ $(8 \pi)^{1 / 2} \sigma \rho^{1 / 2} \mu^{-1} c_{1}^{1 / 2}$, where $\sigma$ is the velocity dispersion of the region, $\mu$ is the ratio of the object's mass-to-flux ratio to the critical value $\left(2 \pi G^{1 / 2}\right)^{-1}, c_{1}$ is a constant of order unity the depends on the cloud's internal density distribution, and $\mu^{-1} c_{1}^{1 / 2} \approx 0.8$ (Basu 2000). This correlation is what one would expect if, in one direction, cloud self-gravity were balanced by magnetic plus turbulent pressure. If we use this correlation in (7), and re-write the relation in terms of the virial parameter $\alpha_{\text {vir }} \equiv 5 \sigma^{2} L /(G M)$, we find that all the dependence on dimensional quantities drops out and we are left with

$$
\mathrm{SFR}_{\mathrm{ff}} \sim 0.01 \alpha_{\text {vir }} .
$$

The uncertainty of this calculation is probably more than an order of magnitude, so we should not pay particular attention to the coefficient, and we will not attempt to place points for magnetic regulation models on Figure 5 Major contributors to the uncertainty are ambiguities in the definition of the length and mass scales $L$ and $M$, the lack of three-dimensional numerical simulations to determine how well equation (17) holds on scales larger than a core collapsing to form a single star system, and the possibility that the cosmic ray ionization rate may vary substantially between galaxies. Perhaps most significantly, equation (6) assumes that ambipolar diffusion in a turbulent medium is not substantially faster than in a quiescent one, an assumption that may well fail (e.g. Heitsch et al. 2004; Nakamura \& Li 2005). If turbulence shortens the ambipolar diffusion time scale so that $t_{\mathrm{AD}} \sim t_{\mathrm{ff}}$, as some simulations suggest it might, then magnetic regulation models would produce a value of $\mathrm{SFR}_{\mathrm{ff}}$ that is too high. Whether this occurs or not is a question that will have to be addressed through threedimensional simulations with self-gravity and non-ideal MHD.

Given these uncertainties, we cannot really say whether the rate of star formation in magnetically subcritical clouds subject to ambipolar diffusion is quantitatively consistent with the observations. However, the lack of dependence of $\mathrm{SFR}_{\mathrm{ff}}$ on any properties but the virial parameter implies that we expect the magnetic $\mathrm{SFR}_{\mathrm{ff}}$ to be roughly the same in all virialized objects, a prediction we can compare to observations. For our observed objects, we know that GMCs with masses $\gtrsim 10^{4}$ $M_{\odot}, \mathrm{HCN}$ clumps, and CS clumps are roughly virialized (Plume et al. 1997; Hever et al. 2001; Wu et al. 2005), so $\alpha_{\text {vir }} \approx 1$ for them. For IRDCs we lack kinematic information from optically thin molecular emission, and therefore we cannot directly determine the velocity dispersion and the virial parameter. If we assume that these objects are virialized, then the magnetic regulation model is broadly consistent with observations that $\mathrm{SFR}_{\mathrm{ff}}$ is roughly constant. We cannot make a stronger statement than this because the magnetic regulation model cannot currently make more specific predictions. Note that it is not critical for this purpose that the objects in question truly be in exact virial equilibrium, since they almost certainly are not. We are using the virial parameter only as a way of parameterizing the strength of the turbulence relative to the strength of gravity, so all that we require is that the objects have virial parameters $\alpha_{\text {vir }} \sim 1$, i.e. that they not be either completely collapsing or completely non-self-gravitating.

\subsection{Virialized Turbulence Models}

A fourth idea to explain the low star formation rate is that turbulence inhibits collapse. This idea has a long history, and we refer readers to Mac Low \& Klessen (2004) for a thorough review. Recently, Krumholz \& McKee (2005) synthesized simulations and analysis of turbulenceinhibited star formation by Klessen et al. (2000) and Vázquez-Semadeni et al. (2003) to derive an estimate that turbulence produces a star formation rate $\mathrm{SFR}_{\mathrm{ff}} \approx 0.014\left(\alpha_{\mathrm{vir}} / 1.3\right)^{-0.68}(\mathcal{M} / 100)^{-0.32}$, where $\alpha_{\mathrm{vir}}$ and $\mathcal{M}$ are the virial parameter and Mach number of a star-forming gas cloud. The estimate is based on a derivation of the fraction of mass that is unstable to collapse in a medium that has the density and velocity structure common to supersonic isothermal turbulence, and is calibrated against simulations. Its uncertainty is probably a factor of a few, stemming from uncertainty in the effects of magnetic fields and from the uncertain approximation $\epsilon_{\mathrm{c}}=0.5$. For GMCs, HCN clumps, and CS clumps, typical values are $\alpha_{\text {vir }} \sim 1-2$ and $\mathcal{M} \sim 20-40$ (Solomon et al. 1987; McKee 1999; Plume et al. 1997; Shirlev et al. 2003; Wu et al. 2005). As with the magentic models, exact virial equilibrium is not required, simply that the kinetic and potential energies be comparable. We plot the predicted value of $\mathrm{SFR}_{\mathrm{ff}}$ for this range of parameters in Figure [5] Given the uncertainties in both the observations and the theoretical calculation, there is reasonable agreement.

Simulations of star formation in turbulent gas for which the turbulent energy is roughly equal to the gravitational potential energy, either because it is driven artificially or because the simulations include feedback from protostellar outflows, also give values of $\mathrm{SFR}_{\mathrm{ff}}$ that are broadly consistent with the data. We show three examples in Figure 5 Li et al. (2004) simulate a periodic box in which the kinetic and turbulent energies are approximately equal and the turbulence is driven to keep the level of turbulence roughly constant. They find a star formation rate of $\mathrm{SFR}_{\mathrm{ff}}=0.079$ at a resolution of $512^{3}$ cells. (Since the simulation uses periodic boundary conditions, the true gravitational potential energy is not well-defined, so we cannot state that the virialization is more than approximate.) The simulations are dimensionless, but they suggest scaling to a typical density of $2 \times 10^{5} \mathrm{~cm}^{-3}$. Similarly, in scale-free simulations with strong turbulent driving, Vázquez-Semadeni et al. (2003) and Vázquez-Semadeni et al. (2005) find SFR ff $_{\text {(2) }}$ 0.01. Li \& Nakamura (2006) find $\mathrm{SFR}_{\mathrm{ff}}=0.067$ in a simulation with a central density of $5 \times 10^{4} \mathrm{~cm}^{-3}$ that includes protostellar outflows from forming stars, which keep the kinetic and gravitational potential energies approximately equal. A number of other simulations in which the turbulence is roughly in virial balance with the gravitational energy produce star formation rates in reasonable agreement with observations over the period in the simulations for which that balance is maintained. If the turbulence is sufficiently strong, simulations can achieve SFR $_{\mathrm{ff}} \sim 0.01$ (e.g. Vázquez-Semadeni et al. 
2003; Tillev \& Pudritz 2004; Vázquez-Semadeni et al. 2005).

We note that $\mathrm{SFR}_{\mathrm{ff}}$ by itself does not appear to provide a way of distinguishing the length scale at which turbulence is driven, since simulations in which turbulence is driven primarily at large scales and ones in which it is driven primarily at small scales both seem capable of producing low values of $\mathrm{SFR}_{\mathrm{ff}}$. Some authors (e.g. Vázquez-Semadeni et al. 2003) emphasize the length scale of the driving as critical to determining whether the turbulence can keep $\mathrm{SFR}_{\mathrm{ff}}$ low, and Krumholz \& McKee (2005) show that the characteristic distinguishing driving that gives $\mathrm{SFR}_{\mathrm{ff}} \sim 1$ from driving that produces $\mathrm{SFR}_{\mathrm{ff}} \ll 1$ is how the Jeans length in the gas compares to the sonic length of the turbulence, defined as the length scale for which the turbulent velocity dispersion equals the sound speed. Other diagnostics, such as the IMF and clustering statistics (e.g. Klessen \& Burkert 2001; Schmeia \& Klessen 2004), the linewidth-size relation of the gas (e.g. Ossenkopf \& Mac Low 2002), and the line luminosity of molecular clouds (Hever \& Brunt 2004) suggest that turbulence in molecular clouds is primarily driven on large scales. In this case Krumholz \& McKee show that the condition distinguishing cases with $\mathrm{SFR}_{\mathrm{ff}} \sim 1$ from those with $\mathrm{SFR}_{\mathrm{ff}} \ll 1$ reduces to the statement that clouds with $\alpha_{\text {vir }} \gtrsim 1$ have low values of SFR $\mathrm{Sf}_{\mathrm{ff}}$, while those with $\alpha_{\text {vir }} \ll 1$ have $\operatorname{SFR}_{\mathrm{ff}} \sim 1$. Thus, the combination of observations of $\mathrm{SFR}_{\mathrm{ff}}$ and those showing that most turbulent energy is on large scales imply that turbulence can only reproduce the observed values of SFR $\mathrm{ff}_{\mathrm{ff}}$ if it is driven strongly enough to produce $\alpha_{\mathrm{vir}} \sim 1$.

\subsection{The Formation Timescale of Clusters}

Another important implication of this work is for the formation timescale of star clusters. The star formation efficiency of clusters, defined as the fraction of the initial gas mass that forms stars over the lifetime of the gas clump, is thought to be $\sim 20 \%-50 \%$ (Kroupa et al. 2001; Lada \& Lada 2003). Since the observational data show that $\mathrm{SFR}_{\mathrm{ff}}$ is at most a few percent, this implies that the cluster formation process must take at least $~$ $10 t_{\mathrm{ff}}$ ( $\sim 5$ crossing times).

The recent observation by Wu et al. (2005) that individual HCN clumps in the Galaxy with IR luminosities $L_{\mathrm{IR}}>10^{4.5} L_{\odot}$ lie on the same $L_{\mathrm{IR}}-L_{\mathrm{HCN}}$ correlation as entire galaxies also provides indirect evidence that the cluster formation time scale is comparatively long. Wu et al. suggest that luminous HCN clumps fall on the galactic IR-HCN correlation because they contain enough stars to sample the IMF fully. Our results showing a large scatter in the light output for small clusters support this conjecture. However, our results on the age-dependence of the luminosity suggest that a mass large enough to sample the IMF is not sufficient by itself to place a cluster on the extragalactic IR-HCN correlation. Since star formation in the galaxies surveyed by Gao \& Solomon (2004a b) has been ongoing for $\gtrsim 3$ Myr, their IR luminosities trace the star formation rate, and the $L_{\mathrm{IR}}-L_{\mathrm{HCN}}$ correlation is therefore a statement of the star formation rate per unit mass in $\mathrm{HCN}$ gas. If the Galactic HCN clumps observed by Wu et al. (2005) typically survived for only $\sim 1$ free-fall time, $\sim 0.2 \mathrm{Myr}$, then as Figure 1shows their luminosity per unit star formation rate would be quite different than that of an older galactic population, and they would not lie on the galactic correlation. One would only expect Galactic HCN clumps to follow the extragalactic $L_{\mathrm{IR}}-L_{\mathrm{HCN}}$ correlation if they are massive enough to sample the IMF well and if they are $\gtrsim 1$ Myr old, which means that they must have existed for at least $\sim 5 t_{\mathrm{ff}}$, and probably closer to $10 t_{\mathrm{ff}}$.

These two lines of evidence provide additional support for the argument that star clusters form in nearequilibrium gas clumps presented by Tan et al. (2006). Tan et al. discuss the implications of this finding in more detail, but we note here two of the most significant. First, formation times of $\sim 10 t_{\mathrm{ff}}$ or longer appear inconsistent with models in which turbulence in protocluster gas is freely decaying. Simulations show that if turbulence is allowed to decay freely (e.g. Bonnell et al. 2003) or never contained energy comparable to the gravitational potential energy (e.g. Vázquez-Semadeni et al. 2003), the star formation process generally ends in only about $2 t_{\mathrm{ff}}$, with $\sim 50 \%$ of the mass in stars. Only if the turbulence is continually driven can there be enough gas left up to $10 t_{\mathrm{ff}}$ for the star formation process to continue (e.g. Vázquez-Semadeni et al. 2003; Li et al. 2004; Li \& Nakamura 2006). The long cluster formation time scales suggested by the observations therefore imply that either the simulations of decaying turbulence are incorrect or (more likely) the turbulence is continually driven. This is consistent with on observations by Williams et al. (2003) and Quillen et al. (2005), and with simulations of protostellar feedback by Li \& Nakamura (2006). We note again that the observations of $\mathrm{SFR}_{\mathrm{ff}}$ alone put no constraints on how or at what scale the turbulence is driven. It can be from internal sources of feedback on small scales, or from a turbulence cascade in which most of the power is on the size scale of an entire GMC.

A second implication is for the mechanism responsible for the stellar initial mass function. Krumholz et al. (2005) have recently emphasized that the competitive accretion mechanism (Bonnell et al. 2006, and references therein) can only operate in the context of a strongly sub-virial gas clump that is undergoing global collapse and converting order unity of its gas mass into stars in a free-fall time. Bonnell \& Bate (2006) concur that the question of whether competitive accretion occurs or not depends on the extent to which gas and stellar velocities in a forming star cluster remain tightly coupled for the majority of the formation process, which in turn depends on whether feedback is effective at driving turbulence, which would change gas velocities but not stellar velocities. If turbulent driving keeps clusters virialized for many free-fall times and limits $\mathrm{SFR}_{\mathrm{ff}}$ to $\lesssim 0.1$, then competitive accretion cannot occur within those clusters. The observations we discuss here provide evidence for low values of $\mathrm{SFR}_{\mathrm{ff}}$ and long cluster formation times, and therefore provide strong evidence against the possiblity that competitive accretion determines the IMF.

\section{CONCLUSIONS}

We present observational evidence for two surprising conclusions, one shown quite strongly and the other more tentatively. First, and very clearly, star formation in dense gas is slow. The time required to convert all the 
gas into stars, the depletion time, is longer than the free-fall time by at least and order of magnitude, and probably closer to two orders of magnitude. There is no evidence that gas at densities up to $n_{H} \sim 10^{5} \mathrm{~cm}^{-3}$ resides primarily in objects that are undergoing collapse and rapid star formation. Second, and more tentatively, the ratio of free-fall time to the depletion time is independent of the characteristic density of the star-forming object in question. The data at present are uncertain, and are insensitive to changes of less than roughly an order of magnitude. Nonetheless, it is interesting that this ratio varies by no more than an order of magnitude from the typical density of GMCs to the typical density of $\mathrm{HCN}(1-0)$-emitting gas, a range of nearly 3 decades in density.

These observations are a strong constraint for theories of star formation, and seem difficult to explain in the context of models in which there is a transition from slow, unbound star formation to rapid, bound star formation somewhere between the GMC scale and the protocluster scale. Slow star formation in cluster-forming gas also implies that clusters require many free-fall times to assemble, as recently argued by Tan et al. (2006) on other grounds. Models in which star formation takes place in virialized objects and is inhibited by strong magnetic fields are qualitatively consistent with the data, and models in which star formation is inhibited by turbulence are both qualitatively and quantitatively consistent with observations.

In the future it would be extremely useful to improve the data on which Figure 5 is based. One way to do this would be to perform detailed studies of other young clusters and obtain data comparable to that for the ONC. This would provide a method of estimating $\mathrm{SFR}_{\mathrm{ff}}$ that is independent of luminosity conversions and does not suffer from concerns about the completeness of galactic surveys. Another improvement in the data could come from an unbiased survey of CS(5-4) emission in the Milky Way or in another galaxy, which would allow us to replace the upper limit on $\mathrm{SFR}_{\mathrm{ff}}$ we derive here with an actual estimate. This would be particularly valuable because the upper limit on $\mathrm{SFR}_{\mathrm{ff}}$ from $\mathrm{CS}(5-4)$ is well above the estimate from $\mathrm{HCN}(1-0)$, even though the densities are not very different. Unbiased CS observations could likely bring down this point.

Another improvement would be extend the data to higher densities. To accomplish this will require observations either of external galaxies or relatively complete surveys of the Milky Way in molecular transitions that trace densities $\gtrsim 10^{6} \mathrm{~cm}^{-3}$. Determining masses from the luminosities in these transitions will probably require high resolution follow-up observations of Galactic sources in optically thin isotopomers so that the luminosity may be correlated against a virial mass. While this is a significant observational challenge, such surveys might make it possible to identify a scale at which star formation transitions from slow to fast, a crucial datum in understanding the star formation process.

We thank N. Evans, C. McKee, and J. Wu for helpful discussions, and the referee, Ralf Klessen, for comments that improved the quality of the paper. Support for this work was provided by NASA through Hubble Fellowship grant HSF-HF-01186 awarded by the Space Telescope Science Institute, which is operated by the Association of Universities for Research in Astronomy, Inc., for NASA, under contract NAS 5-26555 (MRK).

\section{REFERENCES}

Allen, A. \& Shu, F. H. 2000, ApJ, 536, 368

Ballesteros-Paredes, J. \& Hartmann, L. 2006, Revista Mexicana de Astronomia y Astrofisica, submitted, astro-ph/0605268

Barranco, J. A. \& Goodman, A. A. 1998, ApJ, 504, 207

Basu, S. 2000, ApJ, 540, L103

Blitz, L., Fukui, Y., Kawamura, A., Leroy, A., Mizuno, N., \& Rosolowsky, E. 2005, Protostars and Planets V, in press, astro$\mathrm{ph} / 0602600$

Bonnell, I. A. \& Bate, M. R. 2006, MNRAS, 624

Bonnell, I. A., Bate, M. R., \& Vine, S. G. 2003, MNRAS, 343, 413

Bonnell, I. A., Larson, R. B., \& Zinnecker, H. 2006, Protostars and Planets V, in press, astro-ph/0603447

Bourke, T. L., Myers, P. C., Robinson, G., \& Hyland, A. R. 2001, ApJ, 554, 916

Bronfman, L., Casassus, S., May, J., \& Nyman, L.-Å. 2000, A\&A, 358,521

Carey, S. J., Feldman, P. A., Redman, R. O., Egan, M. P. MacLeod, J. M., \& Price, S. D. 2000, ApJ, 543, L157

Ciolek, G. E. \& Mouschovias, T. C. 1993, ApJ, 418, 774

Clark, P. C. \& Bonnell, I. A. 2004, MNRAS, 347, L36

Clark, P. C., Bonnell, I. A., Zinnecker, H., \& Bate, M. R. 2005 , MNRAS, 359, 809

Crutcher, R. M. 1999, ApJ, 520, 706

Desch, S. J. \& Mouschovias, T. C. 2001, ApJ, 550, 314

Dobbs, C. L., Bonnell, I. A., \& Pringle, J. E. 2006, MNRAS, submitted, astro-ph/0602103

Egan, M. P., Shipman, R. F., Price, S. D., Carey, S. J., Clark, F. O., \& Cohen, M. 1998, ApJ, 494, L199+

Elmegreen, B. G. 2000, ApJ, 530, 277

Gao, Y. \& Solomon, P. M. 2004a, ApJS, 152, 63

-. 2004b, ApJ, 606, 271

Goldreich, P. \& Kwan, J. 1974, ApJ, 189, 441

Graciá-Carpio, J., Garciá-Burillo, S., Planesas, P., \& Colina, L. 2006, ApJ, in press, astro-ph/0602339
Hartmann, L., Ballesteros-Paredes, J., \& Bergin, E. A. 2001, ApJ, 562,852

Heiles, C. \& Crutcher, M. 2005, Magnetic Fields in Diffuse HI and Molecular Clouds (Berlin: Springer), in press, astro-ph/0501550

Heitsch, F., Zweibel, E. G., Slyz, A. D., \& Devriendt, J. E. G. 2004, ApJ, 603, 165

Heyer, M. H. \& Brunt, C. M. 2004, ApJ, 615, L45

Heyer, M. H., Carpenter, J. M., \& Snell, R. L. 2001, ApJ, 551, 852

Hillenbrand, L. A. \& Hartmann, L. W. 1998, ApJ, 492, 540

Hoogerwerf, R., de Bruijne, J. H. J., \& de Zeeuw, P. T. 2001, A\&A, 365,49

Huff, E. M. \& Stahler, S. 2006, ApJ, in press, astro-ph/0603138

Iglesias-Páramo, J., Buat, V., Takeuchi, T. T., Xu, K., Boissier, S., Boselli, A., Burgarella, D., Madore, B. F., Gil de Paz, A., Bianchi, L., Barlow, T. A., Byun, Y.-I., Donas, J., Forster, K., Friedman, P. G., Heckman, T. M., Jelinski, P. N., Lee, Y.-W., Malina, R. F., Martin, D. C., Milliard, B., Morrissey, P. F., Neff, S. G., Rich, R. M., Schiminovich, D., Seibert, M., Siegmund, O. H. W., Small, T., Szalay, A. S., Welsh, B. Y., \& Wyder, T. K. 2006, ApJS, 164, 38

Jappsen, A.-K., Klessen, R. S., Larson, R. B., Li, Y., \& Mac Low, M.-M. 2005, A\&A, 435, 611

Kennicutt, R. C. 1998a, ARA\&A, 36, 189

-. 1998b, ApJ, 498, 541

Klessen, R. S. \& Burkert, A. 2000, ApJS, 128, 287

-. 2001, ApJ, 549, 386

Klessen, R. S., Heitsch, F., \& Mac Low, M. 2000, ApJ, 535, 887

Kroupa, P. 2002, in ASP Conf. Ser. 285: Modes of Star Formation and the Origin of Field Populations, ed. E. K. Grebel \& W. Brandner, 86-+

Kroupa, P., Aarseth, S., \& Hurley, J. 2001, MNRAS, 321, 699

Krumholz, M. R. \& McKee, C. F. 2005, ApJ, 630, 250

Krumholz, M. R., McKee, C. F., \& Klein, R. I. 2005, Nature, 438, 332 
Lada, C. J. \& Lada, E. A. 2003, ARA\&A, 41, 57

Leitherer, C., Schaerer, D., Goldader, J. D., Delgado, R. M. G., Robert, C., Kune, D. F., de Mello, D. F., Devost, D., \& Heckman, T. M. 1999, ApJS, 123, 3

Li, P. S., Norman, M. L., Mac Low, M., \& Heitsch, F. 2004, ApJ, 605,800

Li, Y., Mac Low, M., \& Klessen, R. S. 2005a, ApJ, 620, L19

-. 2005b, ApJ, 626, 823

-. 2006, ApJ, submitted, astro-ph/0508054

Li, Z.-Y. \& Nakamura, F. 2006, ApJ, 640, L187

Mac Low, M. \& Klessen, R. S. 2004, Reviews of Modern Physics, 76,125

Matzner, C. D. \& McKee, C. F. 2000, ApJ, 545, 364

McKee, C. F. 1989, ApJ, 345, 782

McKee, C. F. 1999, in NATO ASIC Proc. 540: The Origin of Stars and Planetary Systems, 29

McKee, C. F. \& Tan, J. C. 2002, Nature, 416, 59

-. 2003, ApJ, 585, 850

McKee, C. F. \& Williams, J. P. 1997, ApJ, 476, 144

McKee, C. F., Zweibel, E. G., Goodman, A. A., \& Heiles, C. 1993, in Protostars and Planets III, 327

Menten, K. M., Pillai, T., \& Wyrowski, F. 2005, in IAU Symposium 227: Massive Star Birth: A Crossroads of Astrophysics, ed. R. Cesaroni, M. Felli, E. Churchwell, \& M. Walmsley, 23-34

Mouschovias, T. C., Tassis, K., \& Kunz, M. W. 2005, ApJ, in press, astro-ph/0512043

Mueller, K. E., Shirley, Y. L., Evans, N. J., \& Jacobson, H. R. 2002, ApJS, 143, 469

Nakamura, F. \& Li, Z.-Y. 2005, ApJ, 631, 411

Ossenkopf, V. \& Mac Low, M.-M. 2002, A\&A, 390, 307

Padoan, P., Jimenez, R., Juvela, M., \& Nordlund, Å. 2004, ApJ, 604, L49

Palla, F. \& Stahler, S. W. 1999, ApJ, 525, 772

-. 2000, ApJ, 540, 255

Plume, R., Jaffe, D. T., Evans, N. J., Martin-Pintado, J., \& GomezGonzalez, J. 1997, ApJ, 476, 730

Prantzos, N. \& Aubert, O. 1995, A\&A, 302, 69

Quillen, A. C., Thorndike, S. L., Cunningham, A., Frank, A., Gutermuth, R. A., Blackman, E. G., Pipher, J. L., \& Ridge, N. 2005, ApJ, 632, 941
Rathborne, J. M., Jackson, J. M., Chambers, E. T., Simon, R., Shipman, R., \& Frieswijk, W. 2005, ApJ, 630, L181

Rathborne, J. M., Jackson, J. M., \& Simon, R. 2006, ApJ, in press, astro-ph/0602246

Salpeter, E. E. 1955, ApJ, 121, 161

Schmeja, S. \& Klessen, R. S. 2004, A\&A, 419, 405

Shirley, Y. L., Evans, N. J., Young, K. E., Knez, C., \& Jaffe, D. T. 2003, ApJS, 149, 375

Shu, F. H. 1992, Physics of Astrophysics, Vol. II (University Science Books)

Shu, F. H., Adams, F. C., \& Lizano, S. 1987, ARA\&A, 25, 23

Simon, R., Jackson, J. M., Rathborne, J. M., \& Chambers, E. T. 2006, ApJ, in press, astro-ph/0511079

Solomon, P. M., Rivolo, A. R., Barrett, J., \& Yahil, A. 1987, ApJ, 319,730

Tan, J. C. 2005, in IAU Symposium 227: Massive Star Birth: A Crossroads of Astrophysics, ed. R. Cesaroni, M. Felli, E. Churchwell, \& M. Walmsley, 318-327

Tan, J. C., Krumholz, M. R., \& McKee, C. F. 2006, ApJ, 641, L121

Tan, J. C. \& McKee, C. F. 2002, in ASP Conf. Ser. 267: Hot Star Workshop III: The Earliest Phases of Massive Star Birth, ed. P. Crowther, 267-+

Tasker, E. J. \& Bryan, G. L. 2006, ApJ, in press, astro-ph/0512027

Tassis, K. \& Mouschovias, T. C. 2004, ApJ, 616, 283

Tilley, D. A. \& Pudritz, R. E. 2004, MNRAS, 353, 769

Vázquez-Semadeni, E., Ballesteros-Paredes, J., \& Klessen, R. S. 2003, ApJ, 585, L131

Valdettaro, R., Palla, F., Brand, J., Cesaroni, R., Comoretto, G., Di Franco, S., Felli, M., Natale, E., Palagi, F., Panella, D., \& Tofani, G. 2001, A\&A, 368, 845

Vázquez, G. A. \& Leitherer, C. 2005, ApJ, 621, 695

Vázquez-Semadeni, E., Kim, J., \& Ballesteros-Paredes, J. 2005, ApJ, 630, L49

Williams, J. P., Plambeck, R. L., \& Heyer, M. H. 2003, ApJ, 591, 1025

Wu, J., Evans, N. J., Gao, Y., Solomon, P. M., Shirley, Y. L., \& Vanden Bout, P. A. 2005, ApJ, 635, L173

Zuckerman, B. \& Evans, N. J. 1974, ApJ, 192, L149 